\title{
Liquid Cell TEM and Automated Image Analysis for Nanoparticle Growth Study
}

\author{
Byung Hyo Kim ${ }^{1,2}$, Joodeok Kim ${ }^{1,2}$ and Jungwon Park ${ }^{1,2}$ \\ 1. School of Chemical and Biological Engineering, Institute of Chemical Processes, Seoul National \\ University, Seoul, South Korea. \\ 2. Center for Nanoparticle Research, Institute for Basic Science (IBS), Seoul, South Korea.
}

Nanocyrstals are widely studied for their physical properties and utilized in biology, electronics, optics, and catalyst. However, most of nanocrystal synthesis and their uses are developed empirically with a limited mechanistic understanding. It is mainly because of their size and heterogeneity in structures and physical properties which cannot be easily accessible by conventional analytical methods. Liquid cell TEM (LTEM) has been introduced recently for in-situ study of chemical reactions occurring in liquid [1, 2]. Liquid cells are equipped with microfabricated thin film windows and a vacuum-tight sealing, and thus, allow an opportunity to utilize high spatial and temporal resolution of TEM in studying reactions of colloidal nanoparticles. Fabrication methods of making liquid cells with different types of electron beam transparent window materials including silicon nitride, silicon, and graphene have been introduced [3-7]. Achieving sub-nm spatial resolution by adjusting the thicknesses of window materials and the encapsulated liquid, growth trajectories of different types of nanoparticles have been directly observed $[1,2]$. In many cases, growth trajectories of individual nanoparticles exhibit heterogeneity which cannot be accessible by conventional experimental and theoretical methods. For example, the growth study of Pt nanoparticles by using silicon nitride window LTEM unveiled coexisting populations of different trajectories and critical steps during growth that incorporate non-classical pathways. Following growth trajectories of individual metal nanocrystals, classical growth composed of nucleation and growth takes place concurrently with non-classical growth based on multiple coalescence events of small clusters in the same batch of the reaction while resulting averaged size and shape of nanocrystals are focused [3, 4$]$. In addition, high-resolution observation by using graphene LTEM elucidated that small clusters have preferred orientations when they merge together [4].

However, mechanistic studies of nanoparticle growth in LTEM have been limited to manual tracking of individual nanoparticles in the image series regardless of the type of liquid cell, the size of the field of view, and magnification [3, 4, 6-8]. In this context, it is unavoidable that our understanding relies on a few exemplary observations. Applying automated image analysis to in-situ LTEM image series may solve such problem, but it has been difficult to automate particle tracking because of the low intensity gradient in grayscale TEM image and fluctuation of background contrast in LTEM time series. In this study, we introduce growth mechanism of Pt colloidal nanoparticles understood by computationally analyzing ensemble trajectories observed in in-situ LTEM images.

Silicon nitride window liquid cells are fabricated following microfabrication processes previously reported and used for in-situ LTEM [7, 8]. Precursor solution for the growth is prepared by mixing Pt molecular precursor, surface ligand, and solvent. After loading precursor solution into the silicon nitride liquid cells, they are vacuum sealed and loaded in TEM for real time observation. The electron beam is used for both imaging source and reducing agent to convert $\mathrm{Pt}$ (II) precursors to Pt metallic monomers which is followed by $\mathrm{Pt}$ nanoparticle growth. Irradiating the electron beam onto the solution mixture, TEM time series which includes growth trajectories of hundreds of nanoparticles can be captured for a prolonged time. We apply a combination of image processing algorithm to computationally smooth, 
filter, and eventually binarize grayscale TEM images. Resulting TEM images show nanoparticles with saturated contrast in the background of zero contrast without compromising information with respect to nanoparticle size and shape. By simply counting the number of pixels for nanoparticles in each binarized TEM image, size of individual nanoparticles can be quickly measured and tracked over time along with the number of nanoparticles. It allows us to track growth trajectories of hundreds of nanoparticles without biases that can arise in conventional analysis based on manual tracking.

Temporal map of Pt nanoparticle growth exhibits several distinct stages. In the early stage, two different growth pathways of (i) nucleation and growth and (ii) multiple coalescences events stochastically occurs while nanoparticles focus their sizes as indicated in the decrease both of the number of nanoparticles and of the standard deviation of sizes while they grow. It is followed by the second stage in which size focusing continues without change of the number of nanoparticles. Interestingly, in this stage, nanoparticles with different sizes show different growth rates. Smaller the size of the nanoparticle is, faster it grows, presumably because of the large surface energy of small nanoparticle. This observation is consistent with classical estimation based on Gibbs free energy of nanoparticles which have high surface to volume ratio. Following is the stage where monomers in the solution are mostly consumed and nanoparticles, especially their surfaces, are only contributors to the entire free energy. Small nanoparticles start dissolving to reduce their high surface energy while large nanoparticles grow by uptake of monomers produced from dissolving small ones. As a result, if the growth of nanoparticles is pushed into this limit, they enter to the regime of size defocusing.

In conclusion, we introduce growth mechanism of noble Pt metal nanoparticles that is composed of multiple discrete stages. The proposed growth mechanism is based on the large scale analysis of ensemble trajectories which is enabled by in-situ LTEM of Pt nanoparticle growth and automated image processing to binarize TEM images. Methods we applied in this study can be augmented to in-situ TEM studies of other types of materials including semiconductor, metallic alloy, and heterostructured nanomaterials [9].

\section{References:}

[1] FM Ross, Science 350 (2015), aaa9886.

[2] BH Kim et al, Adv. Mater. 30 (2018), 1703316.

[3] H Zheng et al, Science 324 (2009), p. 1309.

[4] JM Yuk et al, Science 336 (2012), p. 61.

[5] J Park et al, Science 349 (2015), p. 290.

[6] WC Lee et al, J. Phys. Chem. Lett. 8 (2017), p. 647.

[7] BH Kim et al, J. Vis. Exp. 128 (2017), e56335.

[8] J Park et al, ACS Nano 6 (2012), p. 2078.

[9] This work was supported by the National Research Foundation of Korea(NRF) grant funded by the Korea government(MSIT) (No. NRF-2017R1C1B2010434), the National Research Foundation of Korea(NRF) grant funded the Korea government(MSIT) (No. NRF-2017R1A5A1015365), and IBSR006-D1. 